\title{
The implication of the shortage of health workforce specialist on universal health coverage in Kenya
}

\author{
Mumbo Hazel Miseda ${ }^{1 *}$, Samuel Odhiambo Were ${ }^{2}$, Cirindi Anne Murianki ${ }^{3}$, Milo Peter Mutuku ${ }^{4}$ \\ and Stephen N. Mutwiwa ${ }^{5}$
}

\begin{abstract}
Background: Globally, there is an acute shortage of human resources for health $(H R H)$, and the greatest burden is borne by low-income countries especially in sub-Saharan Africa and some parts of Asia. This shortage has not only considerably constrained the achievement of health-related development goals but also impeded accelerated progress towards universal health coverage (UHC). Like any other low-income country, Kenya is experiencing health workforce shortage particularly in specialized healthcare workers to cater for the rapidly growing need for specialized health care (MOH Training Needs Assessment report (2015)). Efficient use of the existing health workforce including task shifting is under consideration as a short-term stop gap measure while deliberate efforts are being put on retention policies and increased production of HRH.
\end{abstract}

Methods: The Ministry of Health $(\mathrm{MOH})$ with support from the United States Agency for International Development-funded FUNZOKenya project and MOH/Japan International Cooperation Agency (JICA) project conducted a country-wide training needs assessment (TNA) to identify skill gaps in the provision of specialized health care in private and public hospitals in 46 out of Kenya's 47 counties between April and June 2015. A total of 99 respondents participated in the TNA. Structured questionnaires were used to undertake this assessment. The assessment sought to determine the extent of skill gaps on the basis of the national guidelines and as perceived by the County Directors of Health $(\mathrm{CDH})$. The questionnaires were posted to and received by all the respondents a week prior to a face-to-face interview with the respondents for familiarization. Data analysis was done using SPSS statistical package.

Results: Overall, the findings revealed average skill gaps on selected specialists (healthcare professional whose practice is limited to a particular area, such as a branch of medicine, surgery, or nursing, especially, one who by virtue of advanced training is certified by a specialty board as being qualified to so limit his or her practice, Free dictionary) at 85 and $62 \%$ when compared to the guideline and as perceived by the CDH respectively. It also revealed that gynecologists exceeded the requirements by 88 and $246 \%$ against the guidelines and as perceived by the CDH respectively.

Conclusion: There is an overall huge gap in health specialists across the 46 counties, and the focus of training should be on the following specialists: cardio-surgeons, neurosurgeons, oncologists, nephrologists, lung and skin clinical officers, anesthetic clinical officers, cardiology nurses, forensic nurses, dental nurses, accident and emergency nurses, and oncology nurses. More innovative approaches, including the use of technology, need to be considered to address this challenge in the immediate, medium, and long terms. Policies and legal frameworks should be developed to facilitate cross-county sharing of specialist expertise. Efforts need to be made to ensure harmonized skill gaps revealed by the guideline and as perceived by the CDHs to inform the development of mitigation strategies.

Keywords: Health workforce, Shortage, Specialists, Training

\footnotetext{
* Correspondence: hazelmmiseda@gmail.com; hazelmmisseda@gmail.com

${ }^{1} \mathrm{MOH} / \mathrm{JICA}$ OCCADEP Project, Nairobi, Kenya

Full list of author information is available at the end of the article
} 


\section{Background}

Globally, the shortage of healthcare workers is projected to reach 12.9 million by 2035. Currently, that figure stands at 7.2 million. The WHO report (2013) at the Third Global Forum on Human Resource for Health indicated that, if not addressed, the shortages identified in the report will have serious implications on the health of billions of people across the world [1]. Although nearly half of the world's population resides in rural areas, only $38 \%$ of the world's nurses and $25 \%$ of the doctors work in these areas [2].

The shortage of healthcare workers is more prevalent in developing countries. The dire shortage of healthcare workers has considerably constrained achievement of health-related Millennium Development Goals (MDGs) [3] and if not addressed may undermine the achievement of health-related Sustainable Development Goals (SDGs).

This shortage of healthcare workers in many countries, including developed countries such as the United Kingdom and United States of America, is attributable to a number of factors such as an increase in chronic diseases and conflict, brain drain in the case of developing countries, and concentration of such workers in urban areas. Resolving the problem of inadequacy of the health workforce will require a united effort by national and international agencies [3].

About 57 countries, the majority being in Africa and Asia, face severe healthcare workers crisis. The World Health Organization (WHO) estimates that at least 2360000 health providers and 1890000 management support workers are required to fill the existing gap.

Healthcare workers are inequitably distributed with severe imbalances between developed and developing countries, with the latter most adversely affected. SubSaharan Africa, with 11\% of the world's population, $24 \%$ of the global disease burden, and only $3 \%$ of the world's health workers [4], faces the greatest challenge.

In Kenya, the total number of the health workers currently employed in the County Departments of Health as well as in the public, faith-based organization (FBO), and private-for-profit health facilities is estimated at 31412 (Training Needs Assessment, 2016) ${ }^{3}$. These numbers are below the required of 138266 healthcare workers as per the Norms and Standards Guidelines by the Ministry of Health.

In order to offer better health services in Kenya, the Health Sector Strategic Plan III (2012-2017) structured service delivery into four main tiers: tier 1, community; tier 2, primary care level; tier 3, county level; and tier 4, national level. The Kenya Health Policy defines eight policy imperatives ${ }^{2}$ including health infrastructure. To guide the development of a robust health infrastructure, the Ministry of Health reviewed and developed Norms and Standards for infrastructure of human resource for health based on the defined population coverage by tiers. Tier 1 covers a population of 5000 people, a dispensary covers 10000 people, a health center covers a population of 30000 people, a primary hospital covers 100000 people, and a secondary care hospital covers a population of one million, while a national teaching and referral hospital covers a population of five million people. It also took into consideration the complexity and the degree of specialized care reflected at each level of the defined tier.

In the wake of devolution which is a form of decentralization that created two levels of government with distinct and complimentary functions ${ }^{1}$ at national and sub-national, health service delivery and to some extent specialized health care became functions of the county government prompting the need to establish the capacity of the counties' human resource requirement to effectively and efficiently deliver these services.

Benchmarking the human resource for health (HRH) capacity with the WHO requirement and against the capacity of the health training institutions in Kenya is considered essential as it enables the government to forecast its workforce, the past inflow, and expected inflow, thus assisting in effective planning and distribution as dictated by needs.

The Ministry of Health and development partners of health in Kenya undertook training needs assessment (TNA) in September 2015, which assessed the overall gap of health specialists as per the standard numbers that have been set as well as the ideal numbers as perceived by the County Directors of Health (Table 1). This assessment sought to identify the health specialists in 94 county referral hospitals. The service domain included surgery, radiology, central sterile services department (CSSD), medical laboratory, ICU, and renal dialysis.

\section{Objective of the TNA}

The objectives of the TNA are to determine the overall gap of specialized healthcare provision at all levels of the health system and to provide recommendations to address these gaps.

\section{Methods}

The Constitution of Kenya (2010) established two levels of government: the national government and the $47 \mathrm{de}$ volved county governments. Under this new arrangement, the national government has been assigned the function of policy, capacity building, and national referral hospitals while the 47 county governments focus on county health delivery. The constitution further introduced health care as a fundamental basic right every citizen must have access to the highest attainable standards. To facilitate realization of this right, the national governments undertook a TNA to establish the capacity of the county governments to deliver on their new mandate. 
Table 1 A summary of the training needs assessment report tables

\begin{tabular}{|c|c|c|c|c|c|c|c|c|c|}
\hline \multicolumn{10}{|c|}{ The training needs assessment report table showing the gap of health workforce specialists in Kenya } \\
\hline $\begin{array}{l}46 \text { counties } \\
\text { analyzed }\end{array}$ & $\begin{array}{l}\text { Total standard } \\
\text { number }\end{array}$ & $\begin{array}{l}\text { Total } \\
\text { employment }\end{array}$ & $\begin{array}{l}\text { Percent } \\
\text { shortage } \\
\text { by standard }\end{array}$ & $\begin{array}{l}\text { Total } \\
\text { ideal } \\
\text { number }\end{array}$ & $\begin{array}{l}\text { Percent } \\
\text { shortage } \\
\text { by ideal }\end{array}$ & $\begin{array}{l}\text { Mean of } \\
\text { employed } \\
\text { staff per } \\
\text { county }\end{array}$ & $\begin{array}{l}\text { Ideal } \\
\text { mean } \\
\text { per county }\end{array}$ & $\begin{array}{l}t \text { test } P \text { value } \\
\text { [comparing } \\
\text { employed } \\
\text { with ideal] }\end{array}$ & $\begin{array}{l}t \text { test } P \text { value } \\
\text { [compare } \\
\text { employed } \\
\text { with standards] }\end{array}$ \\
\hline $\mathrm{CDH}$ & 138266 & 26763 & 81 & 67312 & 60 & 683 & 1599 & $<0.0001$ & $<0.0001$ \\
\hline $\mathrm{MOH}$ & 6428 & 1719 & 73 & 3942 & 56 & 16 & 21 & 0.0291 & $<0.0001$ \\
\hline Anesthetist & 590 & 106 & 82 & 386 & 73 & 2 & 9 & $<0.0001$ & $<0.0001$ \\
\hline ENT surgeons & 285 & 30 & 89 & 205 & 85 & 1 & 4 & $<0.0001$ & $<0.0001$ \\
\hline Ortho surgeons & 285 & 29 & 90 & 188 & 85 & 1 & 4 & $<0.0001$ & $<0.0001$ \\
\hline Cardio surgeons & 10 & 0 & 100 & 120 & 100 & 0 & 3 & $<0.0001$ & 0.0007 \\
\hline Neuro surgeon & 10 & 4 & 60 & 1140 & 100 & 1 & 2 & $<0.0001$ & 0.135 \\
\hline Urolo surgeons & 10 & 3 & 70 & 115 & 97 & 0 & 3 & $<0.0001$ & 0.0365 \\
\hline Pediatrics surgeons & 10 & 8 & 20 & 139 & 94 & 0 & 3 & $<0.0001$ & 0.6461 \\
\hline Neurologists & 275 & 4 & 99 & 114 & 96 & 0 & 2 & $<0.0001$ & 0.135 \\
\hline Plastic surgeons & 10 & 14 & -40 & 141 & 90 & 0 & 3 & 0.0005 & 0.7089 \\
\hline Obstetricians/gynecologists & 560 & 1054 & -88 & 305 & -246 & 2 & 7 & $<0.0001$ & $<0.001$ \\
\hline General internists/physicians & 570 & 1719 & -202 & 3982 & 57 & 36 & 86 & 0.07986 & $<0.001$ \\
\hline Cardiologists & 20 & 1 & 95 & 110 & 99 & 0 & 2 & $<0.0001$ & $<0.001$ \\
\hline Chest specialists & 0 & 1 & 5813 & 5813 & 100 & 0 & 3 & $<0.0001$ & 0.1515 \\
\hline Endocrinologists & 0 & 1 & 95 & 95 & 99 & 0 & 2 & $<0.0001$ & 0.32 \\
\hline Gastro-enterologists & 20 & 4 & 80 & 105 & 96 & 0 & 2 & $<0.0001$ & 0.013 \\
\hline Graduate clinical officers & 4585 & 939 & 80 & 1807 & 48 & 20 & 40 & 0.0849 & $<0.001$ \\
\hline CO ENT & 1375 & 109 & 92 & 490 & 78 & 2 & 11 & $<0.0001$ & $<0.001$ \\
\hline CO lungs and skin & 1885 & 208 & 89 & 532 & 61 & 5 & 12 & $<0.0001$ & $<0.001$ \\
\hline $\begin{array}{l}\text { CO ophthalmology/ } \\
\text { cataract surgery }\end{array}$ & 1080 & 90 & 92 & 338 & 73 & 2 & 8 & $<0.0001$ & $<0.001$ \\
\hline Co pediatrics & 1395 & 135 & 90 & 552 & 76 & 3 & 12 & $<0.0001$ & $<0.001$ \\
\hline Anesthetist nurses & 1630 & 61 & 96 & 696 & 91 & 1 & 15 & $<0.0001$ & $<0.001$ \\
\hline $\begin{array}{l}\text { Accident and emergency } \\
\text { care nurses }\end{array}$ & 2750 & 13 & 100 & 482 & 97 & 0 & 10 & $<0.0001$ & $<0.001$ \\
\hline BSN nurses & 1180 & 1189 & -1 & 2426 & 51 & 26 & 54 & 0.0225 & 0.9905 \\
\hline KRCHN & 41732 & 8612 & 79 & 21635 & 60 & 17 & 494 & $<0.0001$ & $<0.001$ \\
\hline $\begin{array}{l}\text { Community public } \\
\text { health nurses (KECHN) }\end{array}$ & 77544 & 796 & 99 & 1611 & 51 & 47 & 66 & 0.6084 & $<0.001$ \\
\hline Cardiology nurses & 20 & 0 & 100 & 483 & 100 & 0 & 10 & $<0.0001$ & 0.0006 \\
\hline Total nationally & 282525 & 43612 & 85 & 115264 & 62 & & & & \\
\hline
\end{tabular}

The assessment sought to determine the extent of the overall gaps of specialized care delivery as compared to the defined optimal requirement in the national guideline and as perceived by the County Directors of Health who are in charge of service delivery in the counties.

This TNA was conducted under the leadership of the Director of Human Resources Development based at the Ministry of Health at the national level. A Steering Committee with clear terms of reference was constituted by the Director of Medical Services (DMS) to oversee the assessment process.
This study covered all specialist staff at post-basic, which refers to health workers undertaking specialist training after their diploma or bachelor's degree level currently posted in county (Government of Kenya, FBO, and private-for-profit) hospitals, the numbers required for effective service delivery, and the gaps (by facility, facility levels L4 and L5, and county). This TNA considered functions and job responsibilities, rather than occupations, as the basis for determining required competences and training needs. Kenya Essential Package for Health (KEPH) defines, for each intervention, the 
"lowest cadre" expected to carry out the intervention and the lowest facility level. It is however on which specialized professional categories should provide them.

A total of 99 respondents participated in the TNA. These comprised medical superintendents of level 4 and 5 county referral hospitals, health directors of FBO, and chief executive officers (CEO) of private hospitals. The other respondents were directors from the national referral facilities of Moi and Kenyatta Referral Hospitals as well as the medical superintendent of Mathari National Mental Referral Hospital. These respondents were included in the assessment because of the leadership role they play in these key institutions. The others interviewed included the County Directors of Health of 46 counties. The assessment used structured questionnaires that were sent to respondents for familiarization followed by a face-to-face interview by the enumerators.

Primary data was collected by administering seven structured questionnaires to the respondents. Desktop review was conducted for secondary data. The data collection took a month. The Ministry grouped the country into 11 clusters each consisting of 3 to 10 counties depending on the geographical distribution, namely, Lake Basin, Western, North Rift, South Rift, Central, Eastern, North Eastern, Coast, Central, and Eastern regions, which were divided into two clusters each because of the vast landscape within the counties. Each cluster had a supervisor in charge of data collection while each County Department of Health provided two enumerators who collected the data. Collection of data in each county took 2 to 3 days depending on the availability of the respondents. During the assessment, the County Directors of Health were asked to provide the actual number of staff employed by their departments as well as their perceived ideal number of the staffs required based on the workloads.

Enumerators and supervisors were recruited and trained on data collection. The training included familiarization with the questionnaires and instructions on administration of the tool based on the protocol and their job descriptions. A pre-test of the questionnaire was conducted as part of the training. Based on the feedback from the pre-test, the questionnaires were revised and finalized.

\section{Limitation of the study}

The ideal number of health workforce suggested by the County Directors of Health was based on perception and could have had some bias.

\section{Analysis}

Data entry and analysis was done using SPSS statistical package. As a first step, open questions had to be placed into categorical variables. The data entry and analysis process was guided by established standards of monitoring and evaluation. The United States Agency for International Development (USAID)/FUNZOKenya and Japan International Cooperation Agency (JICA) provided technical leadership in data analysis and reporting.

Descriptive analysis was used in computing frequencies, recoding variables, and running cross tabulation. Frequency counts and percentages were the main statistical tools employed in analyzing the data generated through the questionnaires. A non-response to any item of the questionnaires was considered invalid and excluded from the analysis. Data outputs were organized by question and by the variables described in the expected outputs in section 5 (e.g. county, cadre, level of care, subsector).

During the process of report writing, further analysis was carried out as needed. Tables, graphs, and pie charts were created using the analysis outputs. The graphs were used to illustrate frequencies and therefore occurrences of events or activities.

The draft TNA report was shared with the stakeholders at a meeting held between 19 and 20 November 2015 and feedback included in the final report. The stakeholders comprised of $\mathrm{MOH}-\mathrm{HRD}, \mathrm{MOH}$ technical departments, counties, training institutions, and development partners.

\section{Results}

Human Resources for Health Norms and Standards Guidelines stipulate the number of healthcare workers required by cadre at different tiers of the health sector for effective health service delivery in Kenya. This Guideline further provides the basis for rational and equitable distribution of the health workforce across the different tiers/levels of healthcare delivery in the country.

The TNA data were aggregated and analyzed by cadres at national and county levels and compared with the Norms and Standards Guidelines. The specialized staff distribution and availability was further analyzed according to their functions in either direct service delivery or administration.

In general, the findings reveal that on average, specialists' skill gaps range between 85 and $62 \%$ when compared to the Norms and Standards and as perceived by the $\mathrm{CDH}$ respectively. However, on average, gynecologists, plastic surgeons, and Bachelor of Science in Nursing (BSN) have surpassed the required level by more than $100 \%$ when compared with the national guideline. Specifically, gynecologist have exceeded the requirements by 88 and $246 \%$ against the guidelines and as perceived by the $\mathrm{CDH}$ respectively. This finding should inform further investment in training of gynecologist/obstetrician in the medium term. Further in-depth study should be 
undertaken to establish the reason for the high number of gynecologists beyond the recommended levels.

The study also revealed that physicians are 57\% less than the "ideal" number suggested by the County Directors of Health but thrice as much the recommended number by Norms and Standards Guidelines. There is need for further studies to shed more light on the reasons for the variance in shortage across the entire cadre spectrum as revealed by the perception of the County Directors of Health and the national guidelines.

\section{Gaps in specialists \\ Medical officer specialists}

The skill gaps among medical officer specialists is significant when compared to the national guideline and the reported ideal numbers suggested by the County Directors of Health (CDH). Findings of sampled specialty areas are detailed as follows: neurosurgeons had a gap of 100 and $60 \%$ against the guidelines and as suggested by the $\mathrm{CDH}$ respectively; there was no significant difference between the gap as reflected by the $\mathrm{MOH}$ Norms and Standards Guidelines as compared to the perceived number by the $\mathrm{CDH}$ (95\% and 99\%). The shortage of urologist accounted for 70 and $97 \%$ against the Norms and Standards Guidelines and the ideal numbers by the $\mathrm{CDH}$, respectively, while neurologists had a shortage of $99 \%$ against the Norms and Standards Guidelines and $96 \%$ against the ideal numbers by the $\mathrm{CDH}$. Pediatric surgeons had a shortage of $20 \%$ against the Norms and Standards Guidelines and 94\% against the ideal number by the $\mathrm{CDH}$ respectively.

Overall, there is a big variance in the gaps between the Norms and Standards Guidelines and the ideal numbers by the $\mathrm{CDH}$ that need to be interrogated. The cardiologists and endocrinologists both had a shortfall of $99 \%$ of the health workforce against ideal number suggested by the $\mathrm{CDH}$ while the cardiologists had a shortfall of $95 \%$ against the Norms and Standards Guidelines. Endocrinologists like chest specialists are not reflected in the Norms and Standards Guidelines. Gastro-enterologists had a shortfall of $96 \%$ against the ideal number of workforce suggested by the $\mathrm{CDH}$ while the shortage against the Norms and Standards Guidelines is $80 \%$. The ortho surgeons had a shortfall of $90 \%$ healthcare workers against the Norms and Standards Guidelines and 85\% against the ideal number suggested by the $\mathrm{CDH}$. Ear, nose, and throat surgeons had a shortage of $85 \%$ against the ideal number suggested by the $\mathrm{CDH}$ and $89 \%$ against the Norms and Standards Guidelines. And lastly, anesthetists had $82 \%$ shortage of the health workforce against the Norms and Standards Guidelines and 73\% against the ideal numbers suggested by the $\mathrm{CDH}$. It is interesting to note that there is inconsistency in the required number of plastic surgeons against the Norms and
Standards Guidelines in which there are 14 times more plastic surgeons against the ideal number suggested by the $\mathrm{CDH}$ while against the Norms and Standards Guidelines, there is a surplus of four specialists.

The gaps among other healthcare workers who are working closely with medical officers are detailed below.

\section{Clinical officer (medical assistant) specialists}

A sample of specialized skills was analyzed for this cadre. The findings revealed specialist skill gaps among the clinical officers in the country as follows: overall, the gap for clinical officers who had undertaken a post graduate specialized training was $80 \%$ against the national guidelines compared to $48 \%$ against the ideal numbers suggested by the $\mathrm{CDH}$. Specifically, the gap for ear, nose, and throat (ENT) was $92 \%$ against the national guideline compared to $78 \%$ against the ideal numbers suggested by the $\mathrm{CDH}$ while in lungs and skin speciality, the gaps were 89 and $61 \%$ against the guidelines and as perceived by the $\mathrm{CDH}$ respectively. Regarding ophthalmology/cataract surgery, the gap against the guideline was 92 and $73 \%$ as perceived by the $\mathrm{CDH}$. Regarding specialization in pediatrics, gaps at $90 \%$ according to the Norms and Standards Guidelines and at $76 \%$ as perceived by the $\mathrm{CDH}$ were noted.

\section{Nurse specialists}

Skill gaps among the nurses for sampled specialized health service delivery areas were also noted in the following areas: anesthetist nurses at 96\% against the Norms and Standards Guidelines while at $91 \%$ as suggested by the $\mathrm{CDH}$; trauma and emergency care nurses at $100 \%$ against the Norms and Standards Guidelines and at $97 \%$ as suggested by the $\mathrm{CDH}$; on the contrary, BSN has exceeded the Norms and Standards Guidelines by $1 \%$ but in short supply by $60 \%$ as perceived by the $\mathrm{CDH}$; KRCHN has a shortage of $79 \%$ against the guideline while it has a deficit of $60 \%$ according to the $\mathrm{CDH}$; cardiology nurses had a shortage of 99 and 100\% respectively against the Norms and Standards Guidelines and the ideal numbers by the $\mathrm{CDH}$ respectively. There were no nurse oncologists in the counties.

\section{Discussions}

Kenya's specialized healthcare skill gaps pose a major threat to the delivery of specialized health care. The policies and strategies to address these shortcomings need to be an integral part of the health systems' strengthening agenda. This is now more critical if Kenya is to make significant progress towards attainment of universal health coverage, health-related Sustainable Development Goals, and realization of right to health care as envisioned in the Constitution 2010. Ironically, Kenya recently launched a mega $\$ 420$ million state-of-the-art 
medical equipment project across the 47 counties in all the public hospitals with the aim of improving access to specialized healthcare services. The current incongruence on the specialized health workforce and the ongoing upgrading of equipment and infrastructure is a manifestation of weak health system approach to the sector development.

In December 2012, the United Nations General Assembly adopted Resolution A/RES/63/33, giving further political impetus to universal health coverage and recognizing the need for an "adequate, skilled, well trained and motivated workforce." However, the World Health Report (2006)_-"Working together for Health" and several other reports since then have highlighted unacceptable variations in availability, distribution, capacity, and performance of human resources for health and identified countries below a critical threshold of health workers, deemed to be generally necessary to achieve high coverage of essential health services.

The evidence presented here show that Kenya lags behind on the supply of the needed specialist healthcare workers to help her in both the short and medium terms. The UNICEF report "Addressing the Health Worker Shortage: A critical action for improving maternal and new born Health," shortages of skilled healthcare workers arise from many factors. These include underinvestment in training and recruitment, weak incentive structures, systems and mechanisms for healthcare workers, and migration of skilled healthcare workers from developing countries to industrialized nations triggered by the high health worker demand in industrialized countries.

With the coming of devolution, HRH management function was rapidly transferred to counties before appropriate county-level structures and adequate capacity to undertake these functions were in place. There was also lack of clarity over specific roles and responsibilities at county and national government and of key players at each level. Subsequently, health worker strikes and mass resignations were witnessed (Tsofa et al. [5]). The factors triggering this exodus include low pay, poor working conditions, and perceived "hostility" from the new employer, the county government. This disquiet has been felt across the cadres resulting in protracted industrial protests by the nurses late 2016 and part of mid-2017 agitating for better working conditions. While the government was swift to address the nurses' concerns, it was so short lived that they are back to the streets for several months. The doctors' strike extended and almost crippled healthcare services for more than 100 days before finally agreeing on a return-to-work formula.

While Kenya trains majority of its health workforce locally, especially at undergraduate level, a good number obtain their post graduate training overseas. As a requirement, upon returning to the country, they have to undergo local clearance process before registration by their respective regulatory bodies. Records show that The Kenya Medical Practitioners and Dentists Board (KMPDB) had registered around 9000 medical doctors and 1000 dentists over the past 32 years, but only $75 \%$ of these are currently considered "active," having renewed their medical licenses within the past 5 years, and $11 \%$ of the active medical doctors are 61 years of age or older, while an additional 17\% are 51-60 years old, although many will continue working after retirement age.

In an effort to enhance efficiency in HRH management especially healthcare specialists between the national and county governments, the two levels of governments have established institutions and mechanisms to facilitate sharing of the scarce specialized skills. These mechanisms include intergovernmental consultative forums and intercounty HRH forums. The Third Global Forum on Human Resources for Health provided an opportunity for an inclusive dialog with many stakeholders involved in efforts to develop and effectively manage human resources globally and specifically in low-income countries.

Key highlights from the training needs assessment reveal that a total of 31412 healthcare workers are employed in the County Departments of Health, FBOs, and private-for-profit health facilities while the Norms and Standards Guidelines recommend 138266 and the ideal number expected as suggested by the $\mathrm{CDH}$ is 67312 . This is with exception of Baringo County which was however excluded due to logistical concerns. This shows that there is a shortage of $81 \%$ health workforce against the Norms and Standards and 60\% against the ideal numbers suggested by the $\mathrm{CDH}$ at the county level. This means that delivery of Kenya's Essential Package for Health (KEPH) is jeopardized due to inadequate staffing levels especially in counties such as Turkana, Uasin Gishu, Wajir, Kajiado, and Garissa which operate at staffing levels below 10 (Ministry of Health TNA Report [6]). There are no cardiologists, oncologists, and nephrologists employed in the counties. Clinical officers are least employed in Samburu, Nairobi, Wajir, Bomet, and Lamu counties. Nurses specialized in forensics, dentistry, and accident and emergency are the fewest in the counties while there is no nurse specialized in oncology.

\section{Conclusion}

What emerged from this assessment is that Kenya is experiencing a severe shortage of health specialist which is likely to jeopardize her efforts towards realization of rights to health and a threat to the attainment of Sustainable Development Goals (SDGs). Therefore, there is an urgent need for policies and strategies that provide a holistic approach to human resources development and management in the short, medium, and the long terms 
to bridge the existing huge skill gaps especially for the specialists in the 47 counties. The already established mechanisms like the intergovernmental consultative forums, for addressing HRH challenges in the counties, need to be strengthened and scaled up. To be considered in these policies and strategies is the specific roles played by each cadre and the use of technology in the national and health sector development as Kenya strides to join the league of upper middle-income countries. The question of whether development of specialists in Kenya should be left to market forces or whether some level of government intervention needs to be introduced is a debate that needs to be ignited as soon as possible. This debate is at the backdrop of significant investment and over supply of some specialized areas (gynecologists/ obstetricians).

More specifically, the focus on the training of specialists should be cardio-surgeons, neurosurgeons, oncologists, nephrologists, lung and skin clinical officers, anesthetic clinical officers, and cardiology nurses, forensic nurses, dental nurses, accident and emergency nurses, and oncology nurses. On the other hand, from the findings, the least number of health specialists required for effective service delivery are pathologists, epidemiologists, and dermatologist. This should be noted by the Human Resource Development Unit of the Ministry of Health when doing training projections.

Given the disparity in the Norms and Standards Guidelines and perception of the County Directors of Health in the ideal number of specialists required of service delivery, there should be an all-inclusive and rational review of the norms and standards to include the views of the County Directors of Health.

\section{Endnote}

${ }^{1}$ Kenya Law report-Constitution of Kenya, 2010

${ }^{2}$ Kenya Health Policy 2014-20130, Ministry of health, afya house, cathedral road, http://www.health.go.ke

${ }^{3}$ http://www.health.go.ke, Email:ps@health.go.ke

\footnotetext{
Abbreviations

$\mathrm{CDH}$ : County Directors of Health; $\mathrm{CDoH}$ : County Department of Health; DMS: Director of Medical Services; FBO: Faith-based organization; GoK: Government of Kenya; HRD: Human Resource Development; $\mathrm{HRH}$ : Human resource for health; KEPH: Kenya Essential Package for Health; KHSSP: Kenya Health Sector Strategic and Investment Plan; MoH: Ministry of Health; SPSS: Statistical Package for the Social Sciences; TNA: Training needs assessment; UHC: Universal health coverage
}

\section{Acknowledgements}

The TNA exercise was the result of a collaborative effort between the Ministry of Health - Human Resources Development Unit (HRD), County Departments of Health $(\mathrm{CDOH})$, and key stakeholders in health workforce development that include the Transitional Authority (TA), the USAID-funded FUNZOKenya project, JICA-supported "Organisational Capacity Development for the Management of Devolved Health Systems" Project, and DANIDA. We express our appreciation to the Technical Steering Committee that was appointed by the Director of Medical Services (DMS) to plan and implement the TNA on behalf of the Ministry.
Funding

Funding of this research was provided by the Ministry of Health, Kenya, USAID FUNZOKenya Project, and JICA OCCADEP Project.

\section{Authors' contributions}

HMM participated in the development of the concept note for the TNA and tools and the data collection and analysis. She wrote this manuscript and reviewed the manuscript. SOW participated in reorganizing, reviewing, and proofreading the manuscript. CAM participated in the data collection and review of the TNA report and this manuscript. MPM participated in the development of the concept note for the TNA and tools, data collection and analysis, and review of this manuscript. SNM reviewed this manuscript. All authors read and approved the final manuscript.

\section{Ethics approval and consent to participate}

Not applicable.

\section{Consent for publication}

Disclaimer: any part of this document may be freely reviewed, quoted, reproduced, or translated in full or in part, provided the source is acknowledged. It may not be sold or used in conjunction with commercial purposes or for profit.

Report of The Training Needs Assessment of Kenya's Health Workforce, MOH 2015. Published by: Ministry of Health, Afya House Cathedral Road, PO Box 30016, 00100 Nairobi, Kenya Email: ps@health.go.ke http://www.health.go.ke

\section{Competing interests}

The authors declare that they have no competing interest.

\section{Publisher's Note}

Springer Nature remains neutral with regard to jurisdictional claims in published maps and institutional affiliations.

\section{Author details}

${ }^{1} \mathrm{MOH} / \mathrm{JICA}$ OCCADEP Project, Nairobi, Kenya. ${ }^{2}$ The Palladium Group, Afya Pwani Project, Nairobi, Kenya. ${ }^{3}$ Human Resource Development Unit, Ministry of Health, Nairobi, Kenya. ${ }^{4}$ USAID FUNZOKenya Project, IntraHealth International Inc., Nairobi, Kenya. ${ }^{5}$ Jhpiego, Nairobi, Kenya.

Received: 2 September 2016 Accepted: 27 October 2017

Published online: 01 December 2017

\section{References}

1. WHO/3rd Global Forum, www.who.int/workforcealliance/forum/2013/hrh_ commitments/en/.

2. United Nations, Population Facts Department of Economic and Social Affairs Population Division (2010). No. 2010/2/E/Ref.

3. V Bhatt, S Giri and S Koiral (2008). Health workforce shortage: a global crisis. The Internet Journal of World Health and Societal Politics Volume 7 Number 1. http://ispub.com/IJWH/7/1/6202

4. WHO. The world health report 2006-working together for health. Geneva: World Health Organization; 2006.

5. Tsofa, B, Goodman C, Gibson L, Molynewx S (2017) Devolution and its effects on health workforce and commodities management-early implementation experiences in Kilifi County, Kenya International Journal for Equity in Health 2017 16:169 https://doi.org/10.1186/s12939-017-0663-2

6. Ministry of Health (2015). Report of the training needs assessment of Kenya's health workforce, http://www.health.go.ke. Accessed 20 July 2017. 